\title{
Two-pulse measures of temporal integration in the fovea and peripheral retina
}

\author{
MICHAEL D. GOTTLIEB, MITCHELL L. KIETZMAN, and IRA J. BERENHAUS \\ Queens College of the City University of New York, Flushing, New York \\ and New York State Psychiatric Institute, New York, New York
}

\begin{abstract}
The temporal integration of luminous energy was compared in the fovea and at $7^{\circ}$ eccentricity using a white-appearing stimulus provided by a glow modulator tube transilluminating a $21.8^{\prime}$ opal glass target in a free-viewing situation. In Experiment 1, a three-interval temporal forcedchoice adaptive staircase procedure was used to estimate thresholds for brief (3-msec) and long (102-msec) two-pulse stimuli. The data of five observers showed that in the fovea the long stimulus required significantly more luminous energy at threshold than the brief stimulus $[t(14)=5.60$, $\mathrm{p}<.01]$; however, at $7^{\circ}$, threshold luminous energy was not significantly different for the brief and long stimulus. Thus, Bloch's law held in the periphery but not in the fovea, and the critical duration was longer ( $>102 \mathrm{msec}$ ) in the periphery than in the fovea $(<102 \mathrm{msec})$. In Experiment 2, the detectability of a brief ( $3 \mathrm{msec}$ ) and a long (102 msec) two-pulse stimulus, both equal in total luminous energy, was compared at the same two retinal locations using a yes-no signal-detection procedure. The data of three observers showed that in the fovea the detectability of the long stimulus was $30 \%$ less than that of the brief stimulus, whereas at $7^{\circ}$ brief and long stimulus detectability was approximately the same. Thus, both experiments demonstrated a foveal-peripheral difference in two-pulse visual temporal integration, with the fovea evidencing less integration.
\end{abstract}

In visual psychophysics, Bloch's law of temporal integration describes the phenomenon whereby stimuli equal in luminous energy produce the same visual effect regardless of how that energy is distributed in time (Graham, 1965). The longest duration stimulus for which Bloch's law holds is known as the critical duration (Hartline, 1934). In the case of rectangular light pulses, stimulus intensity (I) and duration (T) are interchangeable and reciprocally related such that $I \times T=K$ for a constant response such as $50 \%$ frequency of seeing. IT reciprocity suggests that luminous energy is completely integrated by the visual system:

Several studies have compared temporal integration in the fovea and peripheral retina. Although most of these investigations indicate longer critical durations or more integration for peripheral than for foveal stimulation (Sperling \& Jolliffe, 1965; Dannheim \& Drance, 1971; Ronchi \& Novakova, 1971), one study (Van den Brink \& Bouman, 1954) has shown that temporal integration is independent of retinal locus. The latter study differed from the former in two major respects. First, Van den Brink and Bouman used two-pulse stimuli, whereas the others all used single pulses. Second, Van den Brink and

We thank K. Regnat and R. Simon for the construction and maintenance of the equipment, and B. Bienstock for her critical readings of the manuscript. M. D. Gottlieb is also grateful to O. C. McKenna and $\mathrm{J}$. Wallman for their support in the final stages of this project. Funds to support this research were provided in part by a PSC-BHE grant from CUNY to M. L. Kietzman. Requests for reprints should be sent to M. D. Gottlieb, Biology Department, City College of New York, 138th Street and Convent Avenue, New York, NY 10031.
Bouman used an equal-energy design, whereas the others used an equal-performance design. In the equal-energy paradigm, the total luminous energy of the stimulus was kept constant and response frequency was measured as a function of the time between two equal luminance pulses. The response frequency for two-pulse stimuli equal in luminous energy but of different durations was the same when Bloch's law was operating. In the single-pulse studies, threshold luminance was the dependent variable, and this was determined for different duration stimuli. In this case, complete integration was indicated by $I \times T=K$ for different duration stimuli at threshold.

The purpose of the present study was to see whether two-pulse measures of temporal integration differed in the fovea and peripheral retina. In light of the difference in methodology between one-pulse and two-pulse studies, the present investigation used both an equal-energy and an equal-performance paradigm. Experiment 1 was similar to the one-pulse studies in that thresholds for a brief (and therefore completely integrated) and a long two-pulse stimulus were determined in the fovea and at $7^{\circ}$ eccentricity. If the long stimulus is less than the critical duration, its threshold luminous energy will be the same as that for the brief stimulus at each respective locus. Experiment 2, which was similar to the research of Van den Brink and Bouman (1954), compared the detectability of brief and long two-pulse stimuli that were equal in total luminous energy. If Bloch's law holds, these two stimuli will be equally detectable, and if temporal integration is independent of retinal locus, there will be no difference in brief versus long stimulus detectability in the fovea and at $7^{\circ}$ eccentricity. 


\section{EXPERIMENT 1}

\section{Method}

Three females and two males with normal visual acuity (Snellen 20/20) served as paid observers. The stimulus, a white-appearing circular target subtending a visual angle of $21.8^{\prime}$, was either foveally fixated or viewed $7^{\circ}$ from the fovea on the horizontal meridian. The light source was a glow-modulator tube (Sylvania R1131C) operating at a current of $23 \mathrm{~mA}$ and irradiated by an argon ultraviolet lamp (GE AR4). Light pulses were produced with rise and decay times of about 20 and $2 \mu \mathrm{sec}$, respectively. The glow-modulator's output was measured with a Pritchard photometer (Photo Research Corp.) that was checked for accuracy using a 100-fL standard of luminance (Gamma Scientific 220-1). Kodak Wratten neutral density filters and Tiffen metallic filters were used to manipulate stimulus luminance. A transistorized nine-channel timer (Logical Instruments, Inc.) with an indeterminancy of 1 part in 10,000 controlled the duration of the light pulses, the dark intervals, and the other events within each trial.

The two-pulse stimuli consisted of two identical 1-msec light pulses separated by either brief $(1-\mathrm{msec})$ or longer $(100-\mathrm{msec})$ intervals of darkness.

The observers wore dark-adapting red goggles (Polaroid Corp.) for $30 \mathrm{~min}$ prior to dark adapting $10 \mathrm{~min}$ in a light-tight booth. A three-interval temporal forced-choice staircase procedure was used to estimate threshold luminance, that is, the luminous intensity needed for 67\% correct responses (Bruder et al., 1975). Each trial consisted of the following sequence of events: A click marked the beginning of a trial. After $2 \mathrm{sec}$, three 0.5 -sec tones, separated by $0.5 \mathrm{sec}$, were sounded. These tones marked the observation intervals. The stimulus was presented $0.1 \mathrm{sec}$ after the onset of one of the tones. Following the last tone, the observer indicated, by pressing one of three buttons, which interval he thought contained the stimulus. A "beep" sounded if the response was correct. The stimulus intensity of the next trial depended upon the observer's response and on the specific set of stepping rules used in the adaptive staircase procedure (Gottlieb, 1979).

For three observers, four forced-choice adaptive staircase thresholds were obtained per session, one for the brief and one for the long stimulus at each retinal location. Each threshold required 72 trials. For the other two participants, eight thresholds were obtained per session, two for the brief and two for the long stimulus at each locus. Their staircases were terminated after a criterion level of responding was reached, and this required 32 trials per threshold on the average. Within each session, retinal locus was counterbalanced. Sessions lasted approximately $60 \mathrm{~min}$ and brief rests were given between blocks. Viewing was with natural pupils. Two observers were tested monocularly and three were tested binocularly; the results were the same for both viewing methods. Statistical analyses were based on the data obtained from 15 sessions, the total number collected for all subjects.

\section{Results}

The results of Experiment 1 indicate that the critical duration was briefer in the fovea than at $7^{\circ}$ eccentricity. The evidence for this is shown in Figure 1. Each point represents a comparison of the amount of total luminous energy $(I \times T)$ needed to yield $67 \%$ correct responding for a brief (x-axis) and long (y-axis) stimulus. The data points corresponding to $7^{\circ}$ eccentricity fall above and below the solid $45^{\circ}$ diagonal line, indicating that total luminous energy was approximately the same for both brief (mean $=0.022 \mathrm{~mL} \times \mathrm{msec}, \mathrm{SD}=0.013$ ) and long (mean $=0.026 \mathrm{~mL} \times$ msec, $\mathrm{SD}=0.017$ ) stimuli. The results of a dependent $t$ test indicate that threshold lu-

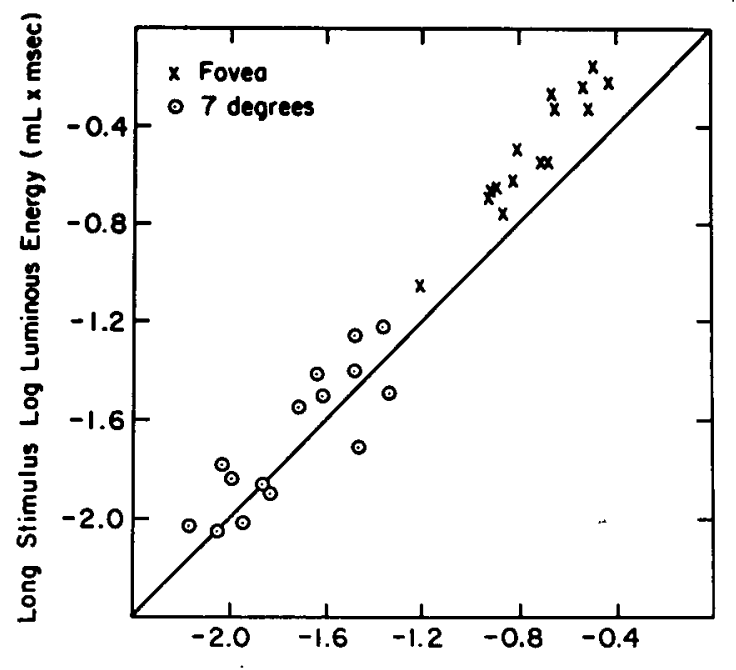

Brief Stimulus Log Luminous Energy ( $\mathrm{mL} \times \mathrm{msec}$ )

Figure 1. Threshold luminous energy for brief and long two-pulse stimuli in the fovea $(x)$ and at $7^{\circ}$ eccentricity $(0)$. Each point represents data from a single testing session. The solid $45^{\circ}$ diagonal line indicates when the brief and long stimuli were of the same total luminous energy. Data points falling above the solid diagonal line indicate that at threshold the long stimulus was of greater total luminous energy than the brief stimulus.

minous energy was not significantly different for the brief and long stimulus $[\mathrm{t}(14)=1.53, \mathrm{p}>.05]$. Therefore, critical duration was longer than $102 \mathrm{msec}$ at $7^{\circ}$ eccentricity. In contrast, the foveal data points fall consistently above the solid diagonal line, indicating that average threshold luminance for the long stimulus was greater than average threshold luminance for the brief stimulus $(0.358 \mathrm{~mL} \times \mathrm{msec}, \mathrm{SD}=0.185$, and $0.199 \mathrm{~mL} \times \mathrm{msec}$, $\mathrm{SD}=0.088$, respectively). The results of a dependent $t$ test support the observation that the two stimuli were different in threshold luminous energy $[t(14)=5.60$, $\mathrm{p}<.01]$. Thus, critical duration was briefer than $102 \mathrm{msec}$ in the fovea. The data of Experiment 1 indicate that the two loci differed with respect to integration. The critical duration was less than $102 \mathrm{msec}$ in the fovea but longer than $102 \mathrm{msec}$ in the periphery.

\section{EXPERIMENT 2}

\section{Method}

Two males and one female from Experiment 1 served as observers. The apparatus was identical to that already described, and the same two retinal loci were stimulated.

The two-pulse stimuli were similar to those used in Experiment 1. However, the luminance of the pulses was not varied; it was set re sensation level at each locus using the brief stimulus. The luminance of the long stimulus was set at the same level. Thus, brief and long stimuli at the same retinal locus were equal in total luminous energy and differed only in the dark interval separating the two pulses.

The observers participated in seven experiments, each consisting of 4 to 16 testing sessions. A session contained eight blocks of trials with retinal locus counterbalanced. Blocks contained an equal number of brief and long stimuli randomized along with 
blanks. Blanks were used to monitor the observer's response criterion and to obtain a measure of signal detectability $\left(\mathrm{d}^{\prime}\right)$ that is independent of response criterion (Green \& Swets, 1966). The number of brief and long stimuli presented at each retinal location varied from 96 to 240 per experiment, and blanks constituted from $20 \%$ to $33 \%$ of all trials per experiment. Trials began with a warning signal followed by a 2-sec foreperiod, after which a stimulus or blank was presented. Dark-adapted observers were instructed to respond "yes" if they thought they saw a light. Participants were tested binocularly, and viewing was with natural pupils. A session lasted approximately $60 \mathrm{~min}$, and brief rests were given between blocks. Statistical analyses were based on the data obtained from the seven experiments.

\section{Results}

The results of Experiment 2 support the findings of Experiment 1 , namely that critical duration was briefer in the fovea than at $7^{\circ}$ eccentricity. In Experiment 2, the dependent measure was response frequency, that is, the percentage of "yes" responses on stimulus trials. Figure 2 shows that response frequency for the brief and long stimulus was similar for the $7^{\circ}$ condition but not for the foveal condition. In the fovea, response frequency was $30 \%$ lower, on the average, for the long stimulus than for the brief stimulus, indicating that critical duration in the fovea was briefer than $102 \mathrm{msec}$. This difference in integration was confirmed statistically using a dependent $t$ test to compare difference scores (response frequency for the brief stimulus minus response frequency for the long stimulus) across retinal locations $[t(6)=4.97$, $\mathrm{p}<.01]$. Thus, the two loci differed with respect to integration, and inspection of Figure 2 shows that the foveal condition displayed less integration.

The false-alarm rate was used as a measure of the observer's response criterion and in the calculation of the

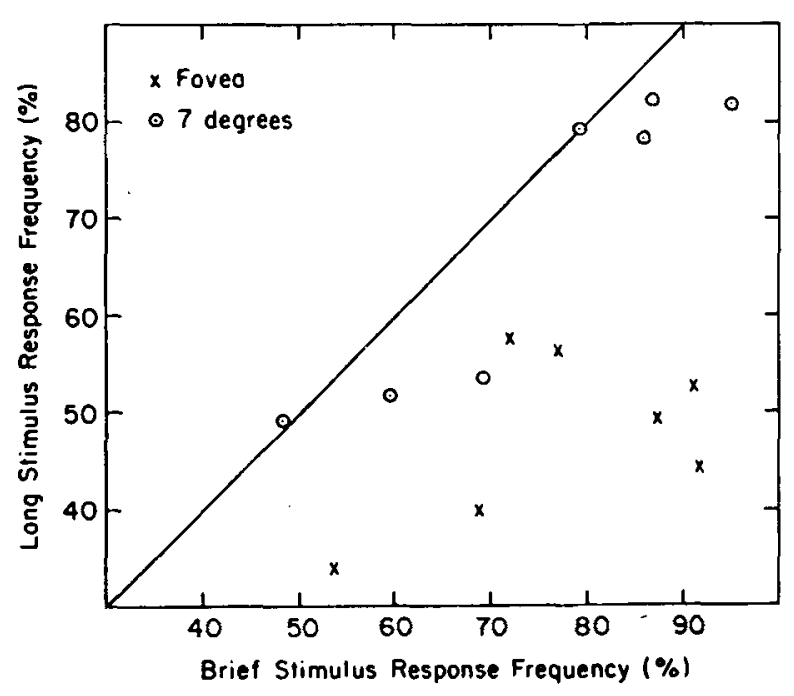

Figure 2. Response frequency for brief and long two-pulse stimuli of the same total luminous energy in the fovea $(x)$ and at $7^{\circ}$ eccentricity $(O)$. Each point represents the data from a single experiment. The solid $45^{\circ}$ diagonal line indicates when the response frequency for the brief and long stimulus was the same. Data points falling below the solid diagonal line indicate that the response frequency for the long stimulus was less than that for the brief stimulus.
Table 1

False Alarm Rate (in Percent)

\begin{tabular}{ccc}
\hline & Fovea & Periphery \\
\hline 9.2 & 5.0 \\
11.2 & 4.2 \\
41.9 & 22.2 \\
18.4 & 31.6 \\
& 15.4 & 11.4 \\
& 11.3 & 10.0 \\
& 19.0 & 10.2 \\
Mean & 18.1 & 13.5 \\
SD & 11.2 & 9.9 \\
\hline
\end{tabular}

signal detectability measure $\mathrm{d}^{\prime}$, which is presumed to be independent of the observer's response bias. Table 1 shows that the false-alarm rate varied considerably across participants and retinal locations, indicating that the observers' response biases differed idiosyncratically. However, the signal detectability measure $\mathrm{d}^{\prime}$ yielded the same results as the frequency-of-seeing measure reported above.

\section{DISCUSSION}

Taken together, the results of the present study indicate that temporal integration differed by retinal locus. Experiment 1 showed that equally detectable brief and long stimuli were of equal luminous energy at $7^{\circ}$ eccentricity but not in the fovea, where the long stimulus required additional luminous energy to reach threshold. Thus, Bloch's law held in the periphery but not in the fovea and the critical duration was longer in the periphery. Experiment 2 showed that brief and long stimuli set equal in total luminous energy differed significantly more in detectability in the fovea than in the periphery. This second experiment confirmed the first experiment in showing more integration in the periphery than in the fovea.

The present finding is consistent with several one-pulse studies that showed longer critical durations or more integration in the periphery than in the fovea (Dannheim \& Drance, 1971; Ronchi \& Novakova, 1971; Sperling \& Jolliffe, 1965). Moreover, our results are in line with numerous investigations of retinal loci with respect to temporal resolution, which can be viewed as the upper limiting case of temporal integration. By implication, these resolution studies lead to predictions about the effects of retinal loci for temporal integration. For example, several studies of the critical flicker fusion (CFF) threshold, the most frequently used measure of temporal resolution, have shown that temporal resolution is greater for foveal than for peripheral stimulation for stimuli less than $30^{\prime}$ in diameter (Hylkema, 1942; Piéron, 1965). Generally, greater temporal resolution upon foveal stimulation implies less temporal integration.

The only two-pulse study to compare temporal integration in the fovea and peripheral retina did not find any difference in integration (Van den Brink \& Bouman, 1954). Thus, this study is the first two-pulse study (using 
two methodologies) to demonstrate a foveal-peripheral difference in temporal integration.

The present research and that of Van den Brink and Bouman (1954) were highly similar. Both used two-pulse stimuli in conjunction with an equal-energy stimulus paradigm to compare temporal integration in the fovea and at $7^{\circ}$ eccentricity. On the other hand, stimulus size and wavelength differed across the two investigations. We used a white-appearing 21.8'-diam stimulus, whereas Van den Brink and Bouman used red and green stimuli $1^{\prime}$ in diameter. Although either of these differences may account for the discrepancy in results, the available evidence points to a complex interaction among stimulus diameter, wavelength, and retinal locus. For example, Sperling and Jolliffe (1965) obtained highly similar integration functions at the fovea and at $15^{\circ}$ eccentricity for a $4.5^{\prime}$ target of 450 and $650 \mathrm{~nm}$ and a $45^{\prime}$ target of $450 \mathrm{~nm}$. However, the function obtained at the fovea using the $45^{\prime} 650-\mathrm{nm}$ target was distinctly different from all the other functions at brief stimulus durations in that deviations from reciprocity were greatest for this stimulus, that is, critical duration appeared to be briefer in the fovea than in the periphery.

Since the criterion that an observer adopts in deciding he has "seen" a stimulus can have a considerable effect upon threshold estimates, prior studies manipulating retinal locus have been criticized for using the classical psychophysical methods of limits and/or constant stimuli, which may have confounded non-sensory (i.e., criterion) and sensory measures (Swets, 1961). The present study overcame this limitation by using a criterion-free forcedchoice procedure (Experiment 1) to measure sensory capacity (Blackwell, 1953; Green \& Swets, 1966) and a yesno signal detection procedure (Experiment 2) from which the measure of sensitivity d' was extracted (Green \& Swets, 1966). Both of these procedures indicated that the critical duration was briefer in the fovea than at $7^{\circ} \mathrm{ec}-$ centricity. Thus, the present finding of a foveal-peripheral difference in temporal integration was independent of the observer's response criterion.

Finally, light adaptation is also known to influence both temporal integration and temporal resolution with greater resolution (higher CFF thresholds) and less integration (shorter critical durations) as the organism is more light adapted (Piéron, 1965). The changes in resolution and integration due to light adaptation may in part be related to changes in the relative balance of rod and cone functioning at different levels of adaptation. Similarly, stimulating different retinal loci may result in a rod-cone balance that can account for the present finding.

\section{REFERENCES}

Blackwell, H. R. (1953). Psychophysical thresholds: Experimental studies of methods of measurement (Report No. 36). Ann Arbor: University of Michigan Press, The Engineering Research Institute.

Bruder, G. E., Sutton, S., Babkoff, H., Gurland, B. K., Yozawitz, A., \& FleIss, J. L. (1975). Auditory signal detectability and facilitation of simple reaction time in psychiatric patients and nonpatients. Psychological Medicine, 5, 260-272.

Dannheim, F., \& DRANCE, S. M. (1971). Studies of temporal summation of central retinal areas in normal people of all ages. Ophthalmological Research, 2, 295-303.

GotTLIEB, M. D. (1979). Two-pulse measures of visual temporal integration in the fovea and at 7 degrees eccentricity (Doctoral Dissertation, City University of New York, 1979). (University Microfilms No. 313130)

GraHAM, C. H. (1965). Some fundamental data. In C. H. Graham (Ed.), Vision and visual perception. New York: Wiley.

GREEN, D. M., \& SWETS, J. A. (1966). Signal detection theory and psychophysics. New York: Wiley.

HARTLINE, H. K. (1934). Intensity and duration in the excitation of single photoreceptor units. Journal of Cellular and Comparative Physiology, 5, 229-247.

Hylkema, B. S. (1942). Examination of the visual field by determining the fusion frequency. Acta Ophthalmologica, 20, 181-193.

PiéroN, H. (1965). Vision in intermittent light. In W. D. Neff (Ed.), Contributions to sensory physiology (Vol. 1; L. M. Hurvich and D. Jameson, Trans.). New York: Academic Press.

RoNCHI, L., \& NovAKOVA, O. (1971). Luminance-time relation at various eccentricities: Individual differences. Journal of the Optical Society of America, 61, 115-118.

SPERLING, H. G., \& JolliFFe, C. L. (1965). Intensity-time relationship at threshold for spectral stimuli in human vision. Journal of the Optical Society of America, 55, 191-199.

SWETS, J. A. (1961). Is there a sensory threshold? Science, 134, 168-177.

VAN DEN BRINK, G., \& BoumAN, M. A. (1954). Variation of integrative actions in the retinal system: An adaptational phenomenon. Joumal of the Optical Society of America, 44, 616-620.

(Manuscript received June 15, 1984; revision accepted for publication December $11,1984$. 